Recebido em 11/2019. Aceito para publicação em 01/2020.

\title{
APLICAÇÃO DE PLANEJAMENTO FATORIAL NA AVALIAÇÃO DA SOLDAGEM A GÁS INERTE DE TUNGSTÊNIO (TIG) EM AÇO MÉDIO CARBONO
}

\section{APPLICATION OF FACTORIAL EXPERIMENTAL DESIGN IN THE TUNGSTEN INERT GAS (TIG) WELDING IN MEDIUM-CARBON STEEL}

\author{
Adyl Hermes Oliveira Contesini ${ }^{1}$ \\ Nathalia Cerqueira da Silva ${ }^{2}$ \\ Elisa Pereira de Carvalho ${ }^{3}$ \\ Rodolfo Salazar Perez ${ }^{4}$ \\ Ana Isabel de Carvalho Santana ${ }^{5}$ \\ Neyda de la Caridad Om Tapanes ${ }^{6}$
}

Resumo: A soldagem a gás inerte de tungstênio (TIG) é um processo de soldagem a arco elétrico entre um eletrodo não consumível de tungstênio e a peça de fusão com proteção gasosa. Atualmente, este processo é amplamente utilizado em metais e ligas de espessura 0,2 $\mathrm{mm}$ e $8 \mathrm{~mm}$. Neste trabalho foi avaliada a soldagem autógena realizada pelo processo automático TIG em cintas metálicas de aço médio carbono utilizadas para embalar barras e feixes de mais de 1 tonelada. Foi utilizado o planejamento fatorial de experimentos para determinar os fatores que influenciam nos ensaios de tração e na microdureza e que qualificam o desempenho das peças soldadas. O modelo de regressão obtido para a carga de ruptura demostrou que o tempo de soldagem é a variável de maior significância estatística, seguida pela corrente. No modelo da microdureza é possível constatar que a variável de maior significância estatística é a variável de blocagem Região soldada (R) seguida do Tempo de solda (Ts) e a Corrente (I).

Palavras-chave: Planejamento de experimentos; soldagem; gás inerte de tungstênio; cintas metálicas; aço médio carbono.

Abstract: Tungsten Inert Gas (TIG) welding is a process of arc welding between a non-consumable tungsten electrode and the gas shielded fusion part, currently widely used in $0.2 \mathrm{~mm}$ - and $8 \mathrm{~mm}$-thickness metals and alloys. In this paper we evaluated the autogenous welding performed by the automatic TIG process on medium carbon steel metal straps used to pack bars and bundles of more than 1 ton. The factorial design of the experiments was used to determine the factors that influence on the tensile tests and on the microhardness and qualify the performance of the welded parts. The regression model obtained for the rupture load showed that welding time is the variable with the greatest statistical significance, followed by current. In the microhardness model, it is possible to verify that the variable with the greatest statistical significance is the blocking variable Welded Region (R) followed by Welding Time (TS) and Current (I).

Keywords: Experiment design; welding; tungsten inert gas; metal belts; medium carbon steel.

\footnotetext{
1 Mestrando do PPCTM, Fundação Centro Universitário Estadual da Zona Oeste, Brasil. E-mail: adyl_contesini@yahoo.com.br.

2 Mestrando do PPCTM, Fundação Centro Universitário Estadual da Zona Oeste, Brasil. E-mail: nathaliacerqueiradasilva@hotmail.com.

3 Mestrando do PPCTM, Fundação Centro Universitário Estadual da Zona Oeste, Brasil. E-mail: elisa.carvalho@sh.com.br.

4 Doutor, Pesquisador, Fundação Centro Universitário Estadual da Zona Oeste, Brasil. E-mail: rsalazarperez@yahoo.com.

5 Doutor, Professor, Fundação Centro Universitário Estadual da Zona Oeste, Brasil. E-mail: isabelcarvalho.uezo@gmail.com.

6 Doutor, Professor, Fundação Centro Universitário Estadual da Zona Oeste, Brasil. E-mail: neydaom@yahoo.com.
} 


\section{INTRODUÇÂO}

Diversas indústrias do segmento de materiais pesados e longos embalam seus produtos com cintas metálicas, que possuem sua fixação através de soldagem. Quando os pontos de solda estão fragilizados podem gerar riscos à segurança e à qualidade ao se realizar a movimentação dos respectivos produtos, seja por ponte rolantes, empilhadeiras ou qualquer outro equipamento. Aárea soldada pode ser prejudicada por diversos motivos, tais como a parametrização de soldagem incorreta e insumos contaminados, gerando defeitos na região soldada, tais como defeitos como porosidade, falta de fusão, micro trincas, podendo estar no interior ou na face do material, logo fragilizando aquela região do material.

Especificamente neste trabalho, foi avaliada a soldagem autógena realizada pelo processo automático TIG (Tungsten Inert Gas) em cintas metálicas de aço médio carbono utilizadas para embalar barras e feixes de mais de 1 tonelada. A soldagem TIG é um processo de soldagem a arco elétrico entre um eletrodo não consumível de tungstênio e a peça de fusão com proteção gasosa, atualmente amplamente utilizado em metais e ligas de espessura $0,2 \mathrm{~mm}$ e $8 \mathrm{~mm}$.

O resultado esperado dessa pesquisa é identificar o parâmetro ideal para o processo de soldagem existente em cintas metálicas utilizadas para embalar, de forma a contribuir para o aumento de produtividade operacional, evitar anomalias no processo de embalagem e mitigar os riscos de segurança ao longo da linha de fabricação.

Para o desenvolvimento deste estudo foi utilizado o planejamento de experimentos fatorial. O planejamento de experimentos é uma ferramenta de engenharia criticamente importante para o desenvolvimento de novos processos ou para melhorar um processo de fabricação. Esta metodologia é empregada para determinar quais parâmetros qualitativos e/ou quantitativos explicam o desempenho de um determinado experimento, buscando, basicamente, satisfazer dois grandes objetivos: a maior precisão estatística possível na resposta e o menor custo. Diversos autores têm utilizado o planejamento de experimentos para avaliar a soldagem TIG.

Kumar e colaboradores (2017) avaliaram a soldagem TIG em aço inoxidável AISI 304 usando um planejamento L27 ortogonal de Taguchi e obtiveram um modelo de regressão linear de segunda ordem. As variáveis de entrada selecionadas foram corrente (60-80 A), tensão (3050 V), abertura de raiz "gap" (0,5-1,5 mm) e a vazão de gás (16-20 l/min). Os valores de resistência à flexão e microdureza foram as variáveis de resposta. A investigação mostrou que a propriedade de dureza é mais afetada pela tensão de soldagem, seguida pela corrente e a abertura da raiz. A análise de superfície de resposta concluiu que a resistência das juntas de solda melhora com a redução da tensão de soldagem e atinge o máximo a 70 ampères de corrente.

Sharma e Singh (2017) publicaram um "review” relacionado à otimização dos parâmetros de soldagem TIG. Foram incluídos trabalhos de vários pesquisadores que 
utilizaram a ANOVA, a metodologia de superfície de resposta (RSM) e outros métodos estatísticos para estudar a otimização da soldagem TIG em aços inoxidáveis. Nas pesquisas, os parâmetros de processo tensão de arco, corrente e taxa de fluxo de gás de proteção foram otimizados. As variáveis respostas foram o efeito simultâneo de parâmetros de soldagem na resistência à tração e na dureza. Verificou-se que a velocidades de soldagem mais baixas, a resistência à tração é influenciada com o aumento da intensidade de corrente.

Moi e colaboradores (2018) investigaram a determinação dos parâmetros de entrada de soldagem de gás inerte de tungstênio (TIG) para obter resistência à tração máxima do aço inoxidável austenítico 316L. Os autores empregaram a metodologia da superfície de resposta Box-Behnken para identificar o efeito dos parâmetros do processo na resistência à tração. Os resultados indicam que a taxa de fluxo de gás tem maior influência na resistência à tração do entalhe, seguida pela corrente de soldagem. As condições otimizadas foram: corrente em 126.77A, velocidade de soldagem em $14.61 \mathrm{~cm} / \mathrm{min}$ e vazão de gás em 8.12 I / min. Também foi determinada a correlação com a microestrutura e a microdureza da solda. O valor da microdureza do metal de solda é notavelmente mais alto do que o da zona HAZ e do metal base. Verificou-se também que quase todas as juntas soldadas exibem maior resistência à tração do que a do metal base.

Shanmugasunda e colaboradores (2019) realizaram experimentos em aço inoxidável austenítico (AISI 304L) usando o processo de soldagem a gás inerte de tungstênio ( $\mathrm{TIG}$ ), no estudo, as juntas soldadas a topo foram feitas usando três níveis de corrente, vazão de gás e bico para distância da peça de trabalho. O arranjo ortogonal L9 do método de projeto experimental de Taguchi foi utilizado para otimizar a corrente de soldagem, a taxa de fluxo de gás e o bocal para a distância da peça de trabalho em juntas soldadas. A condição paramétrica do processo ideal que forneceu os valores mais altos de resistência à tração máxima (UTS) da amostra soldada foram corrente $120 \mathrm{~A}$, taxa de fluxo de gás $10 \mathrm{I} / \mathrm{min}$ e distância do bico à placa $15 \mathrm{~mm}$.

Ahmad e Alam (2019) utilizaram a metodologia da superfície de resposta (RSM) para analisar e representar a causa e o efeito da interação entre respostas e os parâmetros de controle da soldagem a arco de tungstênio a gás (GTAW) numa seção fina de aço inoxidável. Os parâmetros de entrada foram a corrente de soldagem, velocidade, tensão, pulsação no tempo e a resposta foi a profundidade de penetração (DOP). Mediante um projeto composto fatorial central e a aplicação da ANOVA, os autores concluíram que a velocidade de soldagem é o parâmetro mais influente sobre as características de desempenho, sendo a combinação ideal de parâmetros do processo: corrente de soldagem 160, a tensão de soldagem 20 volts, a velocidade de soldagem $193 \mathrm{~mm} / \mathrm{min}$ e o pulso no tempo 45\%. Nesta condição ideal, o DOP previsto foi de $5,53 \mathrm{~mm}$.

A aplicação da metodologia de planejamento de experimento para estudar a 
soldagem TIG em aço carbono também tem sido abordada por diversos autores.

Mohsen, Hamid e Masoud (2016) investigaram a microestrutura e o comportamento do desgaste na superfície do aço carbono. Amostras de aço carbono foram preenchidas através de soldagem TIG aplicando correntes de pulso e contínuas por metal de adição. A fim de otimizar as condições de soldagem e o impacto de cada um dos fatores usados para determinar o grau de dificuldade no modo pulsado, a técnica de desenho experimental de Taguchi e o uso da relação $\mathrm{S} / \mathrm{N}$ foram usados. Por fim, os resultados gerais da análise revelaram que os fatores variáveis, pico de fluxo e baixo fluxo, respectivamente, são considerados os fatores mais influentes na resposta.

Thakur e Chapgaon (2017) publicou um artigo apresentando os efeitos dos parâmetros de soldagem TIG e da soldagem a arco elétrico com eletrodo revestido MMA (Shielded Metal Arc Welding - SMAW). Os fatores de corrente (100-120 A), vazão de gás $(10-15 \mathrm{l} / \mathrm{min})$ e temperatura $\left(25\right.$ a $\left.150^{\circ} \mathrm{C}\right)$ foram utilizados para avaliar a dureza na solda e na zona termicamente afetada (ZTA). A experimentação baseada num planejamento ortogonal Taguchi foi usada para avaliar a soldagem híbrida em material de aço de baixo carbono com processo TIG para passe de raiz e processo MMA para passes subsequentes. No estudo verificou-se que a temperatura possuía elevada influência sobre a dureza da soldadura e a dureza da zona afetada pelo calor. Verificouse também que a dureza da solda foi afetada pela corrente TIG, enquanto a dureza da zona afetada pelo calor foi afetada pela corrente MMA.

O processo de soldagem a gás inerte de tungstênio (TIG) de cordões em barras cilíndricas sólidas de aço EN8 e EN24 foi pesquisado por Hemnani, Mittal e Goyal (2018). As variáveis do processo de entrada, consideradas foram: corrente de soldagem, tensão de soldagem e taxa de fluxo de gás. A significância de cada parâmetro foi estudada usando a ANOVA (Análise de variância). A significância estatística dos parâmetros de entrada foram obtidos mediante a contribuição percentual na resistência à tração, sendo a voltagem $(74,6 \%)$ e a corrente $(18,10 \%)$ os de maior influência na resposta.

Observa-se nestas referências que a corrente de soldagem é uma variável de influência estatística demostrada sobre a soldagem TIG em diversos materiais. Neste contexto o objetivo deste trabalho é avaliar a soldagem no processo automático TIG em aço médio carbono, com vistas a propor um procedimento que evite problemas metalúrgicos na região soldada das cintas metálicas utilizadas para embalagem.

\section{MATERIAIS E MÉTODOS}

O estudo foi dividido em duas partes, a primeira faz uma avaliação dos parâmetros que influenciam nos ensaios de tração e a segunda na microdureza. 


\subsection{Matrizes do Planejamento de Experimentos}

A pesquisa teve como base o planejamento de experimentos realizado mediante o uso do software Statistica'12 para definição da quantidade de amostras a serem retiradas de acordo com as variáveis de soldagem pré-definidas, neste caso, tempo de soldagem (Ts) e corrente (I). Desta forma ficou configurado na Tabela 1 as amostras a serem retiradas de acordo com a combinação dos fatores.

Os fatores citados foram selecionados devido a sua importância e influência no processo de soldagem, além de serem as variáveis de mais fácil acesso e modificação pelo operador do posto de trabalho. As faixas de mínimo e máximo dos fatores foram definidas de acordo com o indicado pelo fabricante do equipamento.

Tabela 1 - Fatores e níveis do planejamento de experimentos.

\begin{tabular}{lccc}
\hline Fatores & $\mathbf{- 1}$ & $\mathbf{0}$ & $\mathbf{1}$ \\
\hline Corrente, $\mathrm{A}(\mathrm{I})$ & 130 & 150 & 170 \\
Tempo de Soldagem, s (Ts) & 1,1 & 1,2 & 1,3 \\
\hline
\end{tabular}

Fonte: Os autores.

As variáveis dependentes escolhidas para quantificar as respostas do experimento foram a carga de ruptura CR para o ensaio de tração e microdureza M para o ensaio de microdureza. Sendo assim, foi realizado para o ensaio de tração um planejamento fatorial completo $22+$ p.c. com 2 fatores e 3 pontos centrais totalizando um total de 7 experimentos para a Carga Ruptura. Para o ensaio de microdureza foi realizado um planejamento fatorial em 6 blocos. Os blocos foram definidos utilizando duas variáveis qualitativas: i) A região de medição da microdureza (variável $R$ ), que foram a poça de fusão, a ZTA e o metal de base; e ii) a solda A ou B (variável S). A Figura 1 mostra a disposição da Solda A e Solda B.

Figura 1 - Indexação das amostras.

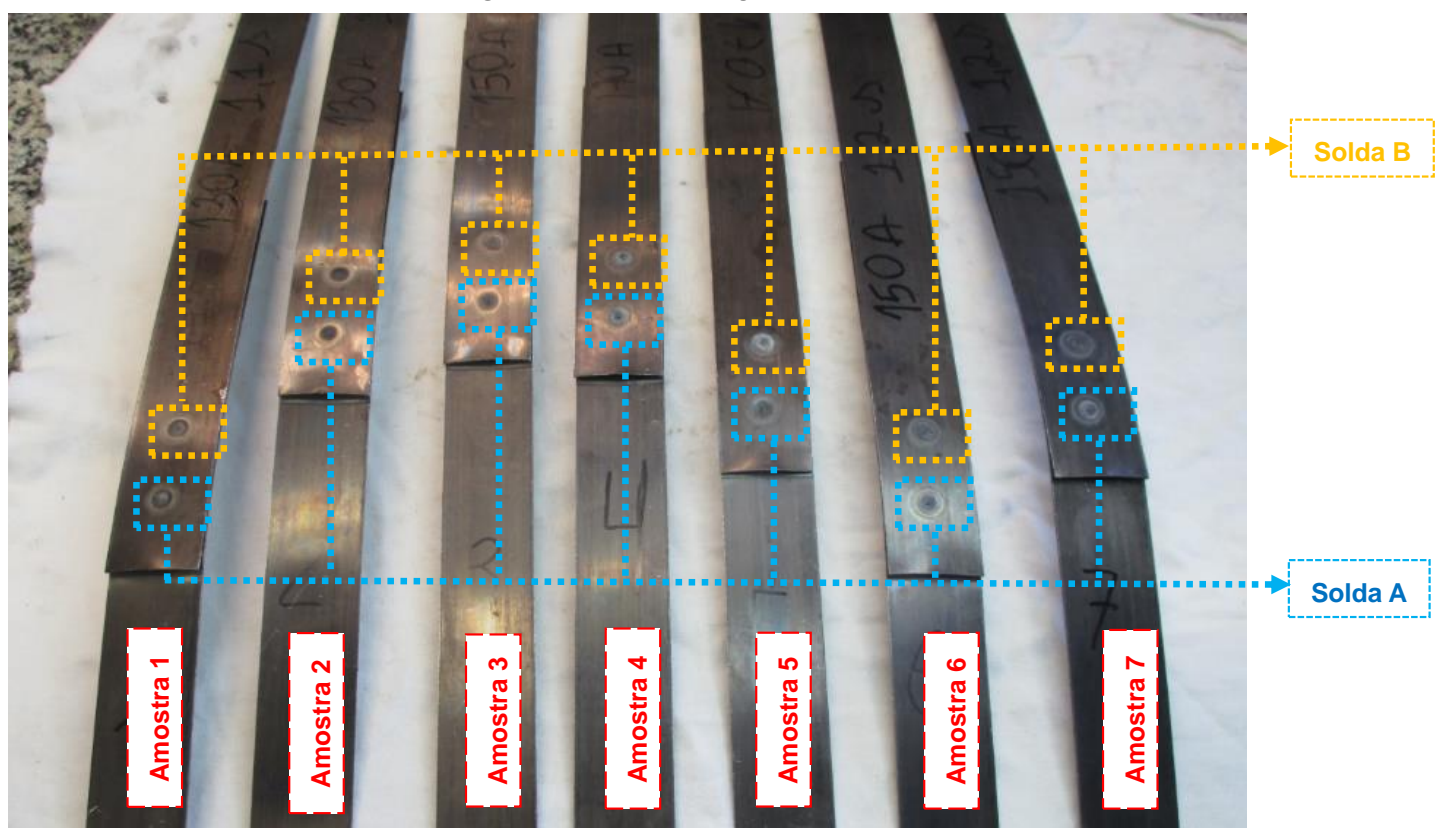

Fonte: Os autores. 


\subsubsection{Ensaio de tração}

A matriz mostrada na Tabela 2 expõe o planejamento de experimentos realizado, o fatorial completo $22+$ p.c. com 2 fatores e 3 pontos centrais totalizando um total de 7 experimentos para a Carga Ruptura.

Tabela 2 - Matriz de planejamento de experimentos para a carga de ruptura do ensaio de tração.

\begin{tabular}{cccc}
\hline Experimentos $^{*}$ & Corrente (I) & Tempo de Soldagem (S) & Carga de Ruptura (CR) \\
\hline 1 & 130 & 1,1 & CR1 \\
2 & 130 & 1,3 & CR2 \\
3 & 170 & 1,1 & CR3 \\
4 & 170 & 1,3 & CR4 \\
5 & 150 & 1,2 & CR5 \\
6 & 150 & 1,2 & CR6 \\
7 & 150 & 1,2 & CR7 \\
\hline
\end{tabular}

${ }^{*}$ Cada experimento representa uma amostra

Fonte: Os autores.

\subsubsection{Microdureza}

Além dos dois fatores considerados na matriz de planejamento da carga de ruptura no ensaio de tração, no ensaio de microdureza devem ser considerados mais dois fatores. Estes são:

- A região de medição da microdureza (variável $R$ ), que foram a poça de fusão, a ZTA e o metal de base; e

- a solda A ou B (variável S)

Ambas variáveis $S$ e $R$ são qualitativas e serão utilizadas como variáveis de blocagem formando 6 blocos de 7 experimentos cada, totalizando 42 (Tabela 3). 
Tabela 3 - Matriz de planejamento de experimentos para a Microdureza (M).

\begin{tabular}{ccccccccccccccc}
\hline Bloco & $\mathbf{I}$ & Ts & $\mathbf{S}^{*}$ & $\mathbf{R}^{\star *}$ & Bloco & $\mathbf{I}$ & Ts & $\mathbf{S}^{*}$ & $\mathbf{R}^{\star *}$ & Bloco & $\mathbf{I}$ & Ts & $\mathbf{S}^{*}$ & $\mathbf{R}^{\star \star}$ \\
\hline & 130 & 1,1 & 1 & 1 & & 130 & 1,1 & 1 & 2 & & 130 & 1,1 & 1 & 3 \\
& 130 & 1,3 & 1 & 1 & & 130 & 1,3 & 1 & 2 & & 130 & 1,3 & 1 & 3 \\
& 170 & 1,1 & 1 & 1 & & 170 & 1,1 & 1 & 2 & & 170 & 1,1 & 1 & 3 \\
1 & 170 & 1,3 & 1 & 1 & 3 & 170 & 1,3 & 1 & 2 & 5 & 170 & 1,3 & 1 & 3 \\
& 150 & 1,2 & 1 & 1 & & 150 & 1,2 & 1 & 2 & & 150 & 1,2 & 1 & 3 \\
& 150 & 1,2 & 1 & 1 & & 150 & 1,2 & 1 & 2 & & 150 & 1,2 & 1 & 3 \\
& 150 & 1,2 & 1 & 1 & & 150 & 1,2 & 1 & 2 & & 150 & 1,2 & 1 & 3 \\
\hline \multirow{4}{*}{2} & 130 & 1,1 & 2 & 1 & & 130 & 1,1 & 2 & 2 & & 130 & 1,1 & 2 & 3 \\
& 130 & 1,3 & 2 & 1 & & 130 & 1,3 & 2 & 2 & & 130 & 1,3 & 2 & 3 \\
& 170 & 1,1 & 2 & 1 & & 170 & 1,1 & 2 & 2 & & 170 & 1,1 & 2 & 3 \\
& 170 & 1,3 & 2 & 1 & 4 & 170 & 1,3 & 2 & 2 & 6 & 170 & 1,3 & 2 & 3 \\
& 150 & 1,2 & 2 & 1 & & 150 & 1,2 & 2 & 2 & & 150 & 1,2 & 2 & 3 \\
& 150 & 1,2 & 2 & 1 & & 150 & 1,2 & 2 & 2 & & 150 & 1,2 & 2 & 3 \\
& 150 & 1,2 & 2 & 1 & & 150 & 1,2 & 2 & 2 & & 150 & 1,2 & 2 & 3 \\
\hline
\end{tabular}

* Solda A - codificada com 1; Solda B - codificada com 2

${ }^{* *}$ Poça de Fusão - codificada com 1; ZTA - codificada com 2; metal base - codificada com 3

Fonte: Os autores.

\subsection{Análise estatística dos resultados do planejamento}

Uma vez definidas as matrizes de experimentos e realizados os respectivos testes de tração e microdureza foram realizadas as análises estatísticas com o software Statitica'12 e obtidos os modelos de regressão de cada variável de resposta. Estes modelos relacionam a variável de resposta com os fatores (Equação 1).

Var. de resposta $=\beta 1^{*}($ Fator 1$)+\ldots+\beta n$ * (Fator $\left.n\right) \pm$ erro

Sendo $\beta 1$.... $\beta$ os coeficientes do modelo de regressão

$\mathrm{Na}$ análise do modelo de regressão foram considerados os modelos sem intercepto (MONTORIL, 2019). Além do modelo de regressão, outras saídas foram obtidas pelo software, estas foram:

- O coeficiente de determinação (R2) que representa a medida de quanto os fatores explicam a variação da variável de resposta. Um ajuste perfeito resulta em $R^{2}=1$.

- O coeficiente de determinação ajustado (R2 ajustado) é o coeficiente múltiplo de determinação $R^{2}$ modificado de modo a levar em conta o número de variáveis e o tamanho da amostra, permitindo remover os erros de multicolinearidade. Ou seja, é uma medida do grau de ajustamento da equação de regressão múltipla aos dados amostrais. 
- A variância residual, $S^{2}$, mede o grau de dispersão entre os valores observados e os estimados da variável de resposta, e a raiz quadrada de $\mathrm{S}^{2}$ é designada erro-padrão da estimativa.

- Estatística F utilizada para testar o efeito conjunto de fatores sobre a variável de resposta. Considerando um nível de significância igual a 0,05; se Significância $F \leq 0,05$, a regressão é significativa, mas se for $>0,05$, a regressão não é significativa (MONTGOMERY, 2005).

- Estatística T representada para cada um dos coeficientes ( $\beta 1$ até $\beta n$ ) tem como objetivo testar se o efeito de cada fator sobre a variável de resposta é ou não estatisticamente significativo. A significância da Estatística T é representada pelo Valorp. Segundo Lapponi (2000) para julgamento compara-se Valor P com o erro tolerado (a) tradicionalmente utiliza-se 0,05.

\subsection{Análise de resíduos}

Esta análise é requerida para validar os modelos de regressão obtidos. Os resíduos numa análise estatística representam o erro, o seja a diferença que existe entre o valor obtido experimentalmente e o calculado pelo modelo, chamemos o primeiro como "observado" e o segundo como "previsto" (MONTGOMERY, 2005).

$\mathrm{Na}$ análise de regressão linear, assumimos que os erros E1, E2, ..., En satisfazem os pressupostos de seguir uma distribuição normal; ter média zero; ter variância constante (homocedasticidade) e ser independentes. Esta análise foi realizada verificando: a normalidade dos resíduos e os diagnósticos de homocedasticidade e independência dos resíduos.

A normalidade dos resíduos é uma suposição essencial para que os resultados do ajuste do modelo de regressão linear obtido sejam confiáveis. Essa condição também pode ser verificada usando um gráfico de probabilidade normal (Normal Probability Plot). O diagnóstico da normalidade dos resíduos será realizado através do gráfico Q-Qplot, este foi construído contrastando o quanti teórico da distribuição normal (z) com os quantis observados a partir do conjunto de dados do estudo (resíduos padronizados e ordenados de forma crescente). Quanto mais próximos os pontos estiverem da reta que representa a distribuição normal, melhor a aderência dos resíduos à distribuição normal.

O diagnóstico de Homocedasticidade será realizado através do gráfico Resíduos padronizados $X$ valores, observados pelo modelo. Se os pontos estão aleatoriamente distribuídos em torno do 0 , sem nenhum comportamento ou tendência, temos indícios de que a variância dos resíduos é homoscedástica. Neste diagnostico também é possível conferir se existem valores extremos (valor atípico ou outlier - uma observação com "alto" resíduo). No trabalho, para definir se uma observação é um "outlier" será analisado se o resíduo padronizado, relacionado a essa observação, for menor que -3 ou maior que 3 , segundo (MONTGOMERY, 2005) esta estimativa corresponde a um intervalo de $99,73 \%$ da distribuição normal padrão. 
Para o diagnóstico de Independência dos resíduos são utilizados métodos gráficos e testes de hipóteses, no trabalho foi utilizado o gráfico de "resíduos padronizados" por "ordem de coleta". Caso os pontos não apresentem nenhum padrão (por exemplo um formato de cone), temos indícios de que a suposição é satisfeita.

\section{RESULTADOS}

\subsection{Planejamento de Experimentos para os ensaios de tração}

A Figura 2 mostra os resultados dos ensaios de tração. A variável Alongamento não foi possível medir, devido às características do material e a forma como foi soldado, sendo a Carga de Ruptura a única variável de resposta deste planejamento de experimentos.

Figura 2 - Resultados dos ensaios de tração, Carga de ruptura (kgf) para a matriz do planejamento.

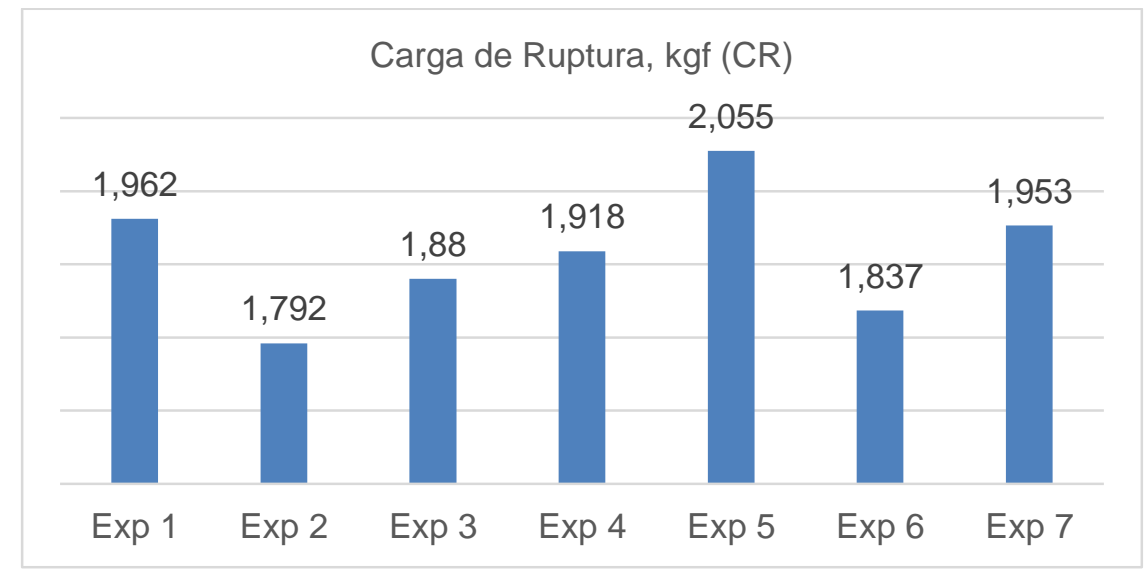

Fonte: Os autores.

As Tabelas 4 e 5 mostram os resultados para a variável de resposta CR. Observase que o modelo de regressão obtido possui um R2 de 0,99 (próximo de 1), um R2 ajustado de 0,79 e a significância $F$ de 0,0000189 (valor < 0,05). Estes resultados sugerem que o modelo pode ser aceito estatisticamente para representar a variável CR na faixa avaliada para os fatores I e Ts.

Tabela 4 - ANOVA para variável carga de Ruptura.

\begin{tabular}{llllll}
\hline & Gl & SQ & $M Q$ & $F$ & Significância $F$ \\
\hline Regressão & 2 & 25,6326 & 8,5442 & 644,5 & 0,0001035 \\
Resíduo & 5 & 0,0530 & 0,0133 & & \\
Total & 7 & 25,6856 & & & \\
\hline
\end{tabular}

Fonte: Os autores.

Analisando o valor-P da Tabela 5, pode-se observar que os fatores Corrente (I) e Tempo de soldagem (Ts) são estatisticamente significativos, pois ambos possuem um valor-p menor que 0,05. Por outro lado, o valor-p da interação I x Ts é maior que 0,05, 
denotando que a influência dela sobre a CR é menor e que não é estatisticamente significativa. No entanto, esta interação por possuir valor-p entre 0,05 e 0,1 ainda pode ser considerada no modelo como um fator marginalmente significativo.

O diagrama de Pareto é uma representação gráfica dos efeitos dos fatores sobre a variável de resposta, a mesma apresenta quais efeitos são relevantes e em que dimensão. Na Figura 3 mostra-se o diagrama de pareto sobre a variável CR corroborando-se que são estatisticamente significativos os fatores I e Ts, sendo este último o de maior influência sobre a Carga de Ruptura no ensaio de tração.

Tabela 5 - Efeitos e coeficientes do modelo de regressão para variável de resposta carga de Ruptura.

\begin{tabular}{llllllll}
\hline & Coeficientes & Erro padrão & Estatística & valor-P & Efeitos & $\begin{array}{l}\text { Inferior } \\
95,0 \%\end{array}$ & $\begin{array}{l}\text { Superior } \\
95,0 \%\end{array}$ \\
\hline Corrente (I) & 0,0146 & 0,0046 & 3,1911 & 0,0332 & 0,0292 & 0,0019 & 0,0273 \\
Tempo de Soldagem (Ts) & 1,4867 & 0,3604 & 4,1254 & 0,0145 & 2,9735 & 0,4861 & 2,4873 \\
Its & $-0,0114$ & 0,0045 & $-2,5565$ & 0,0629 & $-0,0229$ & $-0,0239$ & 0,0010 \\
\hline
\end{tabular}

Fonte: Os autores.

Figura 3 - Diagrama de Pareto do Planejamento Fatorial completo 22 +p.c. da Carga de Ruptura.

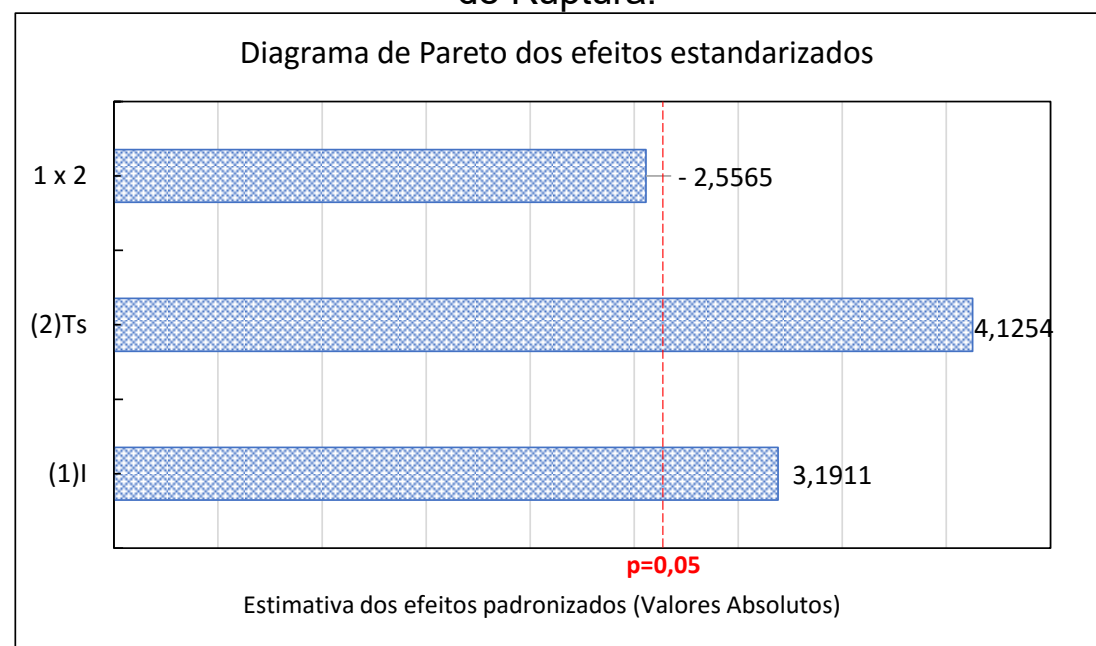

Fonte: Os autores.

Considerando os coeficientes mostrados na Tabela 5 pode-se escrever o modelo de regressão da variável de resposta CR como segue na Equação 2:

$C R=0,0146 * \mid+1,4867^{*} T s-0,0114{ }^{*}{ }^{*} T s \pm 0,1151 \quad$ sendo $R^{2}$ ajustado $=74,69 \%(2)$

A Figura 4 mostra o gráfico de contorno da relação entre as variáveis Corrente, Tempo de Soldagem e Carga de Ruptura. A variação na resposta é principalmente afetada pelo tempo de soldagem, corroborando a discussão anterior. Considerando os valores mostrados no gráfico podemos dizer que, na faixa estudada, os menores valores de tempo de soldagem e os maiores valores de corrente maximizam o valor da Carga 
de ruptura. Resultados similares foram obtidos por Sharma e Singh (2017).

Figura 4 - Gráfico de Contorno do modelo de regressão da Carga de Ruptura.

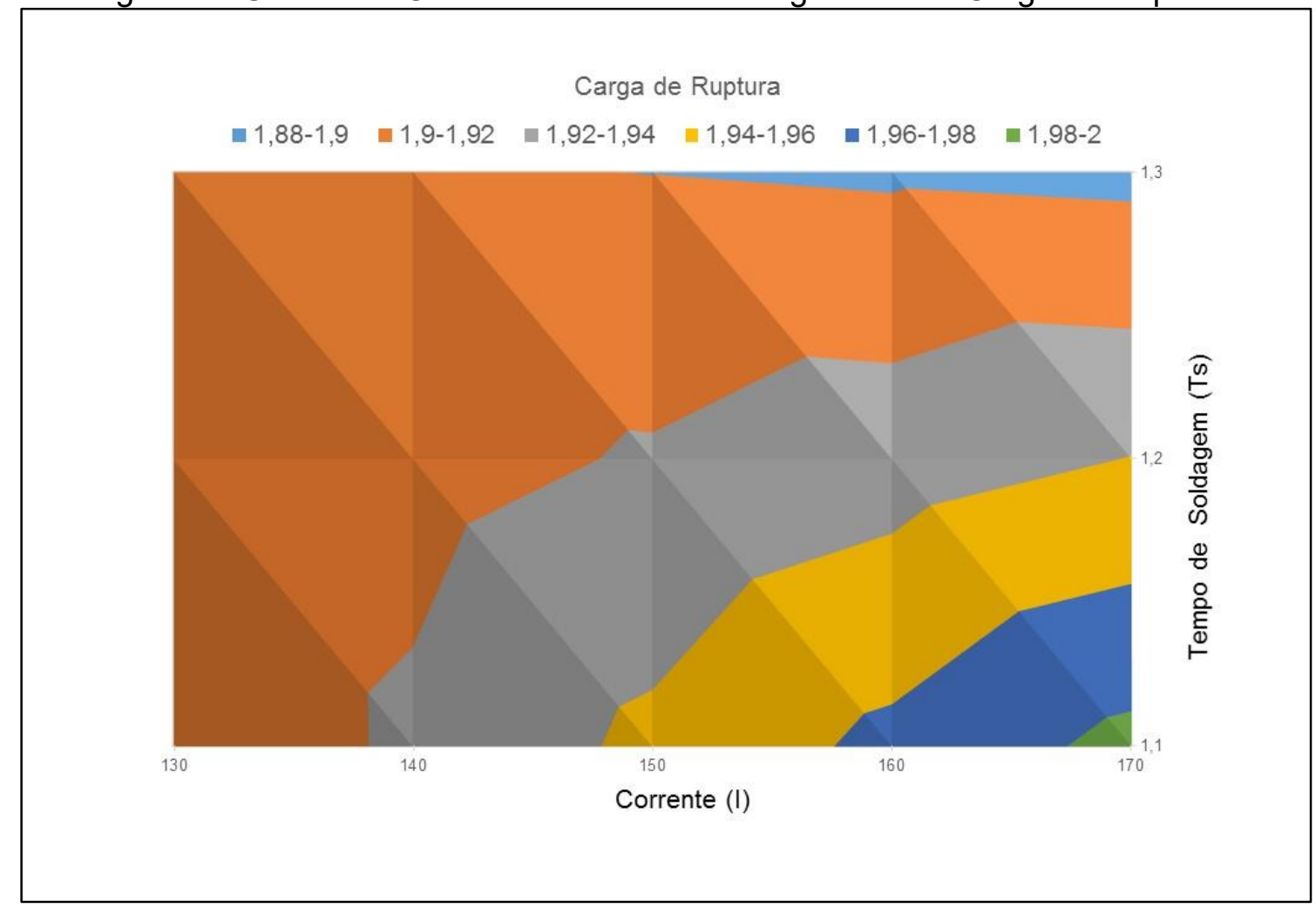

Fonte: Os autores.

\subsection{Resultados do Planejamento de experimentos para os ensaios de Microdureza}

Os resultados do planejamento fatorial em blocos para a análise da variável de resposta Microdureza mostra-se na Figura 5.

Figura 5 - Resultado do ensaio de Microdureza nos experimentos em blocos.

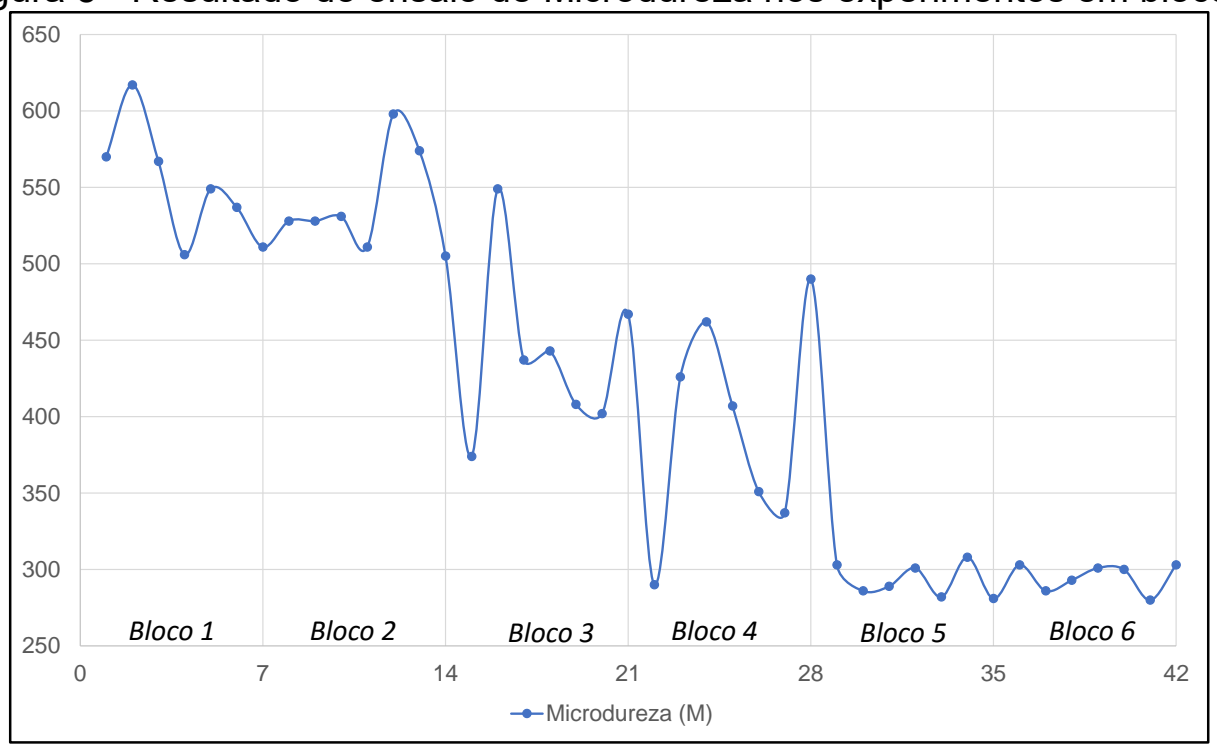

Fonte: Os autores. 
Tabela 6 - ANOVA para variável de resposta Microdureza.

\begin{tabular}{llllll}
\hline & $g l$ & $S Q$ & $M Q$ & $F$ & Significância $F$ \\
\hline Regressão & 5 & 7819376,681 & 1563875,336 & 891,3809 & $1,16388 \mathrm{E}-36$ \\
Resíduo & 37 & 64914,3194 & 1754,4411 & & \\
Total & 42 & 7884291 & & & \\
\hline
\end{tabular}

Fonte: Os autores.

Tabela 7- Efeitos e coeficientes do modelo de regressão para variável de resposta Microdureza.

\begin{tabular}{lrrrrrrrr}
\hline & Coeficientes & $\begin{array}{l}\text { Erro } \\
\end{array}$ & padrão & \multirow{2}{*}{ Estat. $t$} & valor-P & Efeitos & Erro efe. & \multicolumn{2}{l}{$\begin{array}{l}\text { Inferior } \\
95,0 \%\end{array}$} & \multicolumn{2}{c}{$\begin{array}{l}\text { Superior } \\
95,0 \%\end{array}$} \\
\hline Corrente (I) & 4,062 & 0,698 & 5,813 & 0,000 & 2,646 & 0,698 & 5,478 & 2,6465 \\
Tempo de Soldagem (Ts) & 591,013 & 57,38 & 10,29 & 0,000 & 474,731 & 57,38 & 707,295 & 474,731 \\
Solda (S) & $-18,664$ & 12,922 & $-1,444$ & 0,157 & $-44,847$ & 12,922 & 7,518 & $-44,847$ \\
Região (R) & $-125,784$ & 7,914 & $-15,893$ & 0,000 & $-141,820$ & 7,914 & $-109,749$ & $-141,820$ \\
Its & $-3,445$ & 0,678 & $-5,076$ & 0,000 & $-4,8202$ & 0,678 & $-2,070$ & $-4,820$ \\
\hline
\end{tabular}

Fonte: Os autores.

Os resultados da ANOVA mostram que o modelo de regressão possui um bom ajuste. Este resultado foi verificado com R2 ajustado e a significância F: R2 ajustado está próximo de 1 demostrando que existe aderência da equação de regressão aos dados amostrais, a significância $\mathrm{F}$ é praticamente zero o que demostra que a regressão obtida é significativa (Tabela 6).

Analisando os valores-P da Tabela 7 é possível verificar que todas as variáveis $\mathrm{e}$ a interação ITs são estatisticamente significativas com exceção da variável de blocagem Solda (S). A variável $S$ possui um elevando valor-P $(0,1571>\alpha)$ o que significa que existem $15,71 \%$ de probabilidade de que a variável $S$ não represente adequadamente o modelo. Os resultados da ANOVA estão de acordo com os do gráfico de Pareto (Figura 6). Nele é possível constatar que a variável de maior significância estatística é a variável de blocagem Região soldada $(R)$ seguida do Tempo de solda, a Corrente (I) e finalmente a interação IxTs.

Figura 6 - Diagrama de Pareto do Planejamento Fatorial em blocos da Microestrutura.

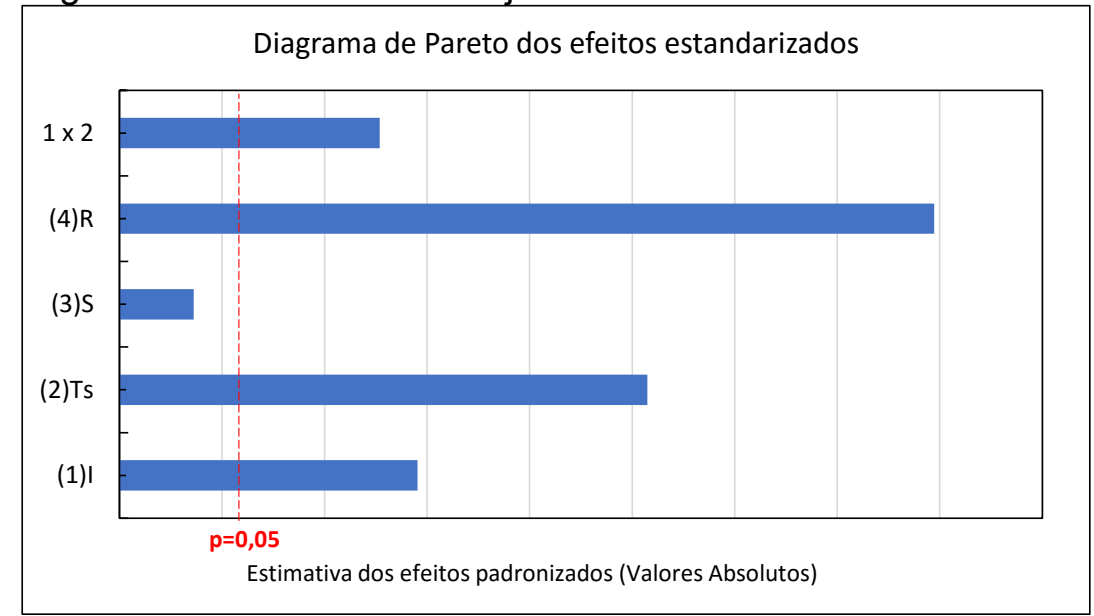

Fonte: Os autores. 
Considerando os coeficientes mostrados na Tabela 7 pode-se escrever na Equação 3 o modelo de regressão da variável de resposta M.

$M=4,06 * I+591^{*} T s-3,44{ }^{*} I^{*} T s-125,78 R \pm 41,78 \quad$ sendo $R^{2}$ ajustado $=96,38 \%(3)$

sendo que a variável de blocagem $R$ é qualitativa e assumirá valores discretos (1 para a região de Poça de Fusão; 2 para a ZTA e 3 para a metal base).

Sendo a variável $\mathrm{R}$ qualitativa podem ser gerados 3 superfícies de resposta representados na Figura 7 com os gráficos de contorno para a região Poça de Fusão, para ZTA e para o Metal base.

Figura 7 - Gráficos de Contorno I-Ts X M para as regiões a) Poça de Fusão, b) ZTA e c) Metal base.

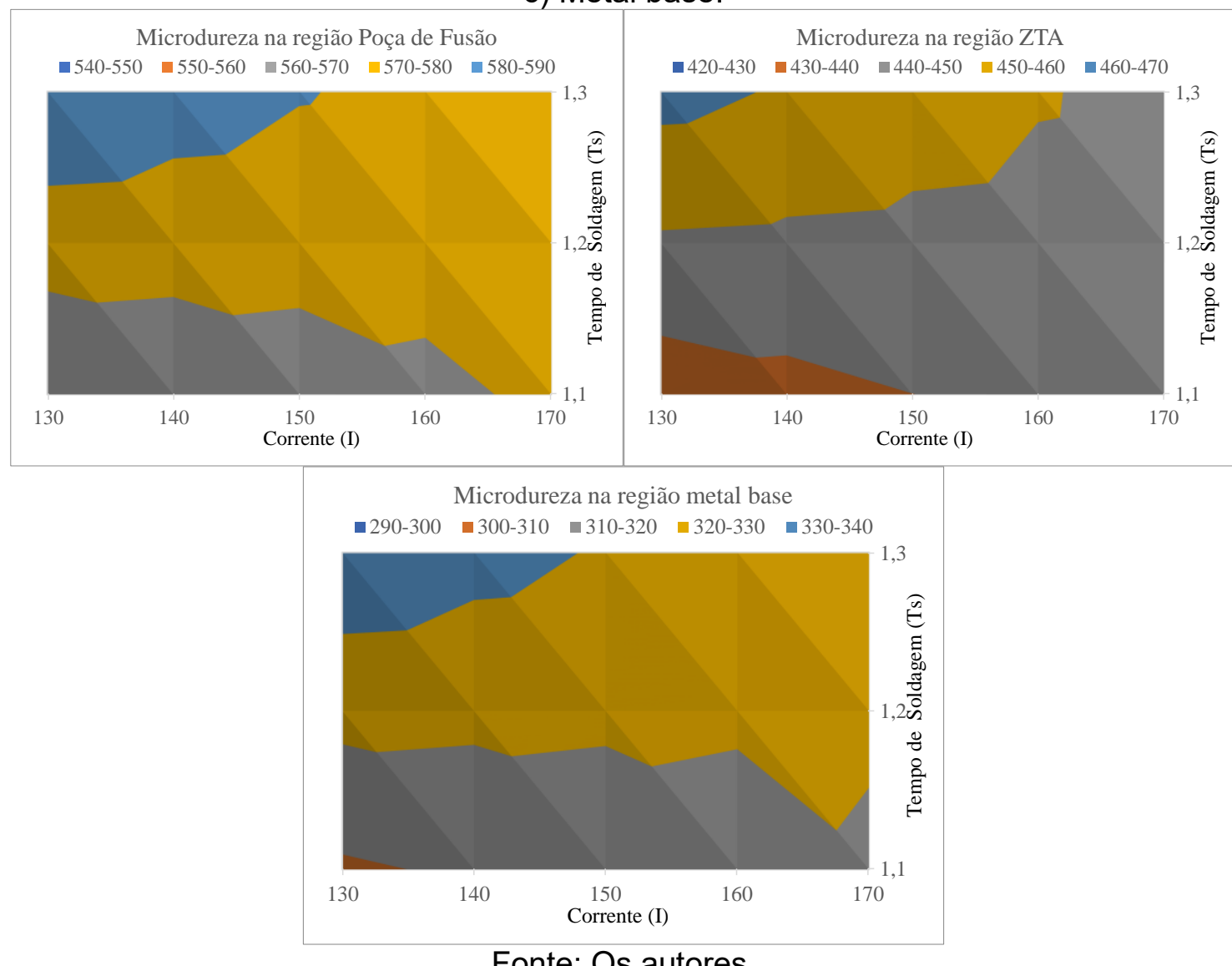

Fonte: Os autores.

Em todos os gráficos, a área sombreada de azul representa a de maior valor de Microdureza, observa-se que ela é atingida quando se utilizam baixos valores de corrente e altos tempos de soldagem. Com a aplicação da metodologia de superfície de resposta se corroboram os resultados da ANOVA, isto é, existe uma forte dependência entre as variáveis microdureza $(M)$ e região soldada $(R)$. Também é possível verificar que a microdureza da poça de fusão é maior que a do ZTA e está maior que a do metal base.

O resultado da fusão pode gerar várias condições ao material fundido, no fim do processo proveniente de um procedimento de soldagem. As partes mais quentes da 
poça de fusão estão localizadas logo abaixo do arco elétrico, logo após a interrupção do arco elétrico a temperatura cai e ocorre a solidificação. Toda solda por fusão acumula um gradiente térmico no metal de base, o qual é influenciada diretamente pela temperatura da poça de fusão e pela velocidade de soldagem. Normalmente, em um ponto da ZTA, logo na borda da poça de fusão, é possível verificar que a temperatura aumenta rapidamente a um nível próximo do da poça de fusão e diminui rapidamente, produzindo um efeito como o do tratamento térmico conhecido como têmpera, que após a evolução e queda de temperatura torna-se um constituinte duro conhecido como martensita. Este comportamento também é encontrado na ZTA, o qual por sua vez também sofre aporte térmico e resfriamento. Logo, é esperado que a região soldada (poça de fusão e zona termicamente afetada) apresente os resultados na Figura 7, o qual demonstra que essa determinada área possui uma dureza superior ao do metal de base (aço médio carbono).

\subsection{Análise dos resíduos dos modelos de regressão das variáveis CR e M}

O modelo de regressão obtido para as variáveis CR (Eq.2) e M (Eq. 3) devem ser validados através de uma análise de resíduos.

\subsubsection{Diagnóstico de Normalidade dos resíduos padronizados}

No Gráfico Q-Q Plot apresentado na Figura 8 verifica-se que os pontos tendem a concentrar-se em torno da reta de declive 0,93 e 0,979 para os modelos de carga de ruptura e microdureza respectivamente. Estes valores podem ser considerados próximos de 1, o que representa que os erros são normalmente distribuídos e a relação empírica foi desenvolvida corretamente em ambos planejamentos.

Figura 8 - Gráfico de normalidade dos resíduos (Q-Qplot) para o modelo de regressão de a) Carga de Ruptura e da b) Microdureza.
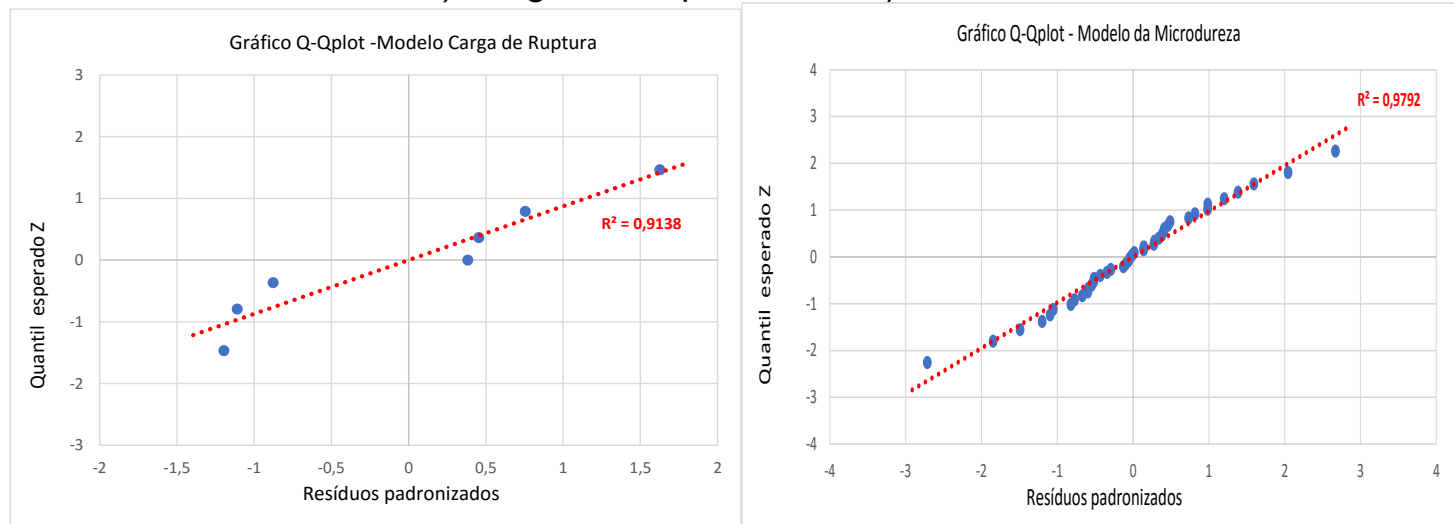

Fonte: Os autores. 


\subsubsection{Diagnóstico de Homocedasticidade}

A Figura 9 mostra os gráficos dos valores previstos por cada modelo de regressão $\mathrm{X}$ : os resíduos padronizados. Observa-se nos gráficos que os resíduos estão distribuídos de forma aleatória em torno à reta, desenhada em resíduos padronizados = 0 . Este resultado dá indícios de ausência de autocorrelação dos resíduos e por tanto que a variância dos resíduos é homoscedástica.

Figura 9 - Valores CR previstos e M previstos pelo modelo X Resíduos Padronizados em cada planejamento.
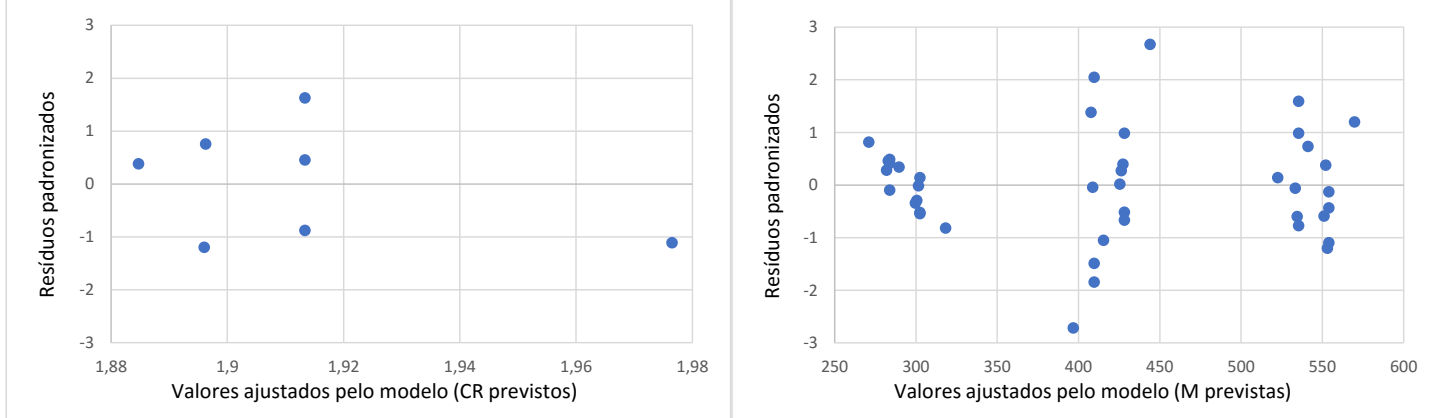

Fonte: Os autores.

Segundo a análise dos resíduos padronizados, nenhum deles possui valor maior que 3 ou menor que -3 , pelo que podemos inferir que não existem pontos extremos (outliers).

\subsubsection{Diagnóstico de Independência}

A Figura 10 mostra o Gráfico de Resíduos padronizados X Ordem de coleta com o objetivo de demostrar a independência dos resíduos do planejamento realizado. Este pressuposto se cumpre pois observa-se no gráfico que os pontos não possuem tendência.

Figura 10 - Resíduos padronizados X Ordem de Coleta (Variável de Resposta CR e M).
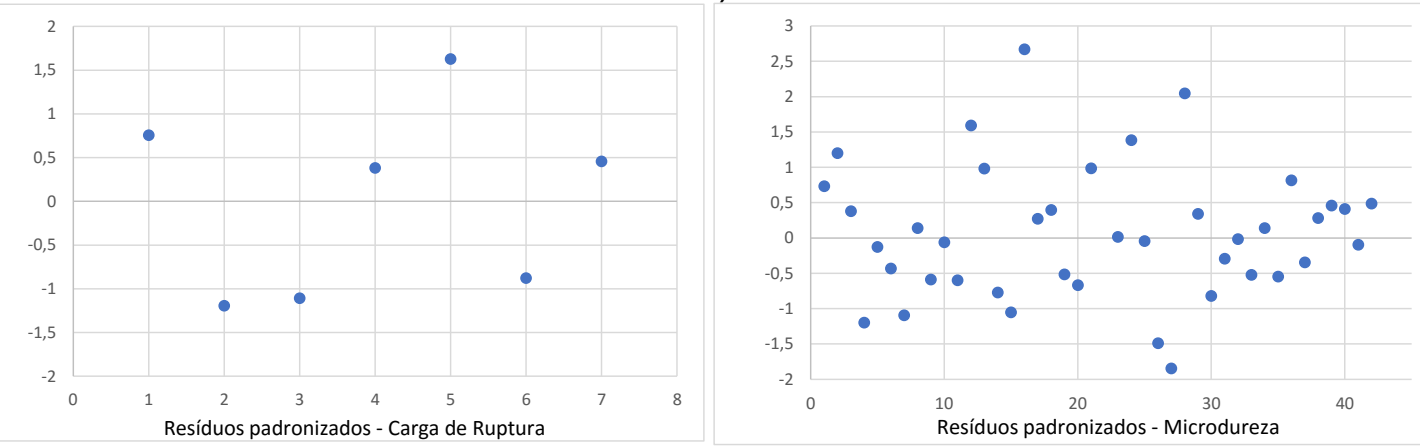

Fonte: Os autores. 


\section{CONCLUSÃO}

- A aplicação do planejamento de experimentos no estudo possibilitou avaliar o desempenho das fitas soldadas pela metodologia TIG, definindo os parâmetros que influenciam nos ensaios de tração e microdureza.

- Por meio dos resultados estatísticos de regressão foi possível corroborar os resultados encontrados na literatura para os ensaios de microdureza. No entanto, devese destacar que a aplicação de um modelo de regressão linear não permite verificar a possível existência da curvatura na superfície de resposta.

- Mediante uma análise dos resíduos, foram validados os modelos de regressão obtidos e a análise dos gráficos de contorno possibilitou definir os parâmetros otimizados de corrente e tempo de soldagem para cada região.

- Considerando que foi utilizada uma modelagem de regressão linear, é recomendável que para trabalhos futuros seja realizado o planejamento de experimentos e a coleta de dados visando a realização do estudo estatísticos de regressão quadrática, a fim de possuir maiores variações nas correlações entre as variáveis e os resultados físicos, aumentando o range de assertividade de resultados esperados.

\section{REFERÊNCIAS}

AHMAD, A.; ALAM, S. Parametric optimization of TIG welding using Response Surface Methodology. Materials Today: Proceedings, v 18, n. 7, p. 3071-3079, 2019.

KUMAR, S. et al. Optimization of TIG Welding Process Parameters Using Taguchi's Analysis and Response Surface Methodology. International Journal of Mechanical Engineering and Technology, v. 8, n.11, p. 932-941, 2017.

HEMNANI, M.; MITTAL, P.; GOYAL, S. Optimization of Tungsten Inert Gas welding using Taguchi and ANOVA. International Journal for Research in Applied Science \& Engineering Technology, v. 6, n. 5, 2018.

LAPPONI, J. C. Estatística Usando Excel 5 e 7. São Paulo: Lapponi, Treinamento e Editora, p. 294, 2000.

MOHSEN, J.N.; HAMID, G.; MASOUD, K. Optimization of pulsed GTAW for hard facing on St37 based with Nob73G2 filler metal. Advanced Processes in Materials Spring, v. 10 , n. 1 (36), p.137-147, 2016.

MOI, S. C. et al. Determination of tungsten inert gas welding input parameters to attain maximum tensile strength of $316 \mathrm{l}$ austenitic stainless steel. Journal of Mechanical Engineering, v. 68, n. 3, p. 231-248, 2018.

MONTGOMERY, D. C. Design and analysis of experiments. 6th. ed. New York: John Wiley \& Sons, 2005.

MONTORIL, M. H. Análise de Regressão: EST036. Regressão sem intercepto. UFJFBrasil. Disponível em: 
http://www.ufff.br/michel montoril/files/2016/05/File09 EST036.pdf. Acesso em: 09 abr. 2019.

SHANMUGASUNDAR, G. et al. Optimization of Process Parameters in TIG Welded Joints of AISI 304L -Austenitic Stainless Steel using Taguchi's Experimental Design Method. Materials Today: Proceedings, v. 16, p. 1188-1195, 2019.

SHARMA, K.; SINGH, I. Optimization of gas tungsten arc welding on steel - A review. International Journal of Interdisciplinary Research, v. 3, n. 1, 2017.

THAKUR, P.; CHAPGAON, A. N. Effect of GTAW-SMAW hybrid welding process parameters on hardness of weld. International Journal of Engineering Research and Technology, v. 10, n. 1, 2017. 